\title{
Chemistry Content in the Pharmacy Curriculum: Relevance to Develop Pharmacists Fit-to-work in Diverse Pharmacy Profession Sectors
}

\author{
Chun Wai Mai ${ }^{*}$, Ramadas Anitha ${ }^{2}$, John Jeh Lung Tiong ${ }^{3}$, Pei Kuan Lai ${ }^{4}$, Mallikarjuna Rao Pichika ${ }^{1}$ \\ and Alexander Irvine Gray ${ }^{5}$
}

${ }^{1}$ Department of Pharmaceutical Chemistry, School of Pharmacy, International Medical University, 126, Jalan Jalil Perkasa 19, Bukit Jalil, 57000, Kuala Lumpur, Malaysia.

${ }^{2}$ Department of Pharmacy, Kuala Lumpur Hospital, Jalan Pahang, 50586, Kuala Lumpur, Malaysia.

${ }^{3}$ School of Pharmacy, Taylor's University, No. 1 Jalan Taylor's, 47500 Subang Jaya, Selangor, Malaysia.

${ }^{4}$ Institute for Research, Development and Innovation. International Medical University, 126, Jalan Jalil Perkasa 19, Bukit Jalil, 57000,

Kuala Lumpur, Malaysia.

${ }^{5}$ Strathclyde Institute of Pharmacy and Biomedical Sciences, University of Strathclyde, 161 Cathedral Street, Glasgow G4 ORE,

Scotland, United Kingdom.

\begin{abstract}
Background: The high employability of pharmacy graduates across various sectors such as in the community pharmacies and hospital settings, the pharmaceutical industries and academia has lent credence to the versatility of their professional training. Therefore, the aim of this study was to gauge the perception of International Medical University (IMU) pharmacy graduates on the applicability and relevance of the chemistry knowledge and practical skills acquired from their professional education in their working place. Methods: The survey was designed based on the pharmacy curriculum of IMU. Qualitative and quantitative responses were collected and analysed by three independent investigators who were not the respondents, and not lecturing in any part of the Chemistry curriculum. The study was completed when the qualitative feedback from respondents reached saturation. Results: Almost equal numbers of pharmacists from the different pharmacy disciplines: (1) industrial and regulatory, (2) hospital and clinical, (3) community and (4) academia, participated in this study. Most of the chemistry topics were rated as essential by the graduates. Some topics were rated very relevant (more than $80 \%$ ) whereas only up to $30 \%$ of the graduates agreed on the essentiality of some topics to their profession. Conclusion: The study concluded that most of the current chemistry content is still essential in nurturing competent pharmacists for their respective disciplines. The outcomes of this study will be used as evidence to support the need for conducting a curriculum review, to better prepare graduates for their future employment.
\end{abstract}

Key words: Chemistry, Competency, Fit-to-work, Graduates, Pharmacy Curriculum, Pharmacy Profession.

Key Messages: Pharmacists operate in diverse employment sectors with different duties and, inter alia, it is clear that knowledge in the subjects of Chemistry is a major requirement for them to perform their daily tasks.

\section{INTRODUCTION}

Many studies are focused on the development of teaching and learning models of Chemistry for pharmacy students. ${ }^{1-5}$ All of these innovative approaches are needed to train competent pharmacists for the ever demanding pharmaceutical services. The planning of an outcome-based curriculum has always been challenging to any pharmacy institution. This is because a thorough up-to-date systematic planning is required to cover all the essential educational outcomes as well as to establish a conducive
Submission Date : 30-03-2015 Accepted Date : :25-05-2015

DOI: $10.5530 /$ ijper.49.4.1 Correspondence Address Dr. Chun Wai Mai Department of PharmaceuticalChemistry,

School of Pharmacy, International Medical University, 126, Jalan Jalil Perkasa 19, Bukit Jalil, 57000 Kuala Lumpur, Malaysia. E-mail:chunwai_mai@imu. edu,mai.chunwai@gmail. com

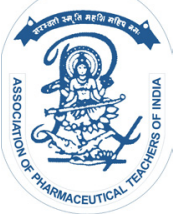

www.ijper.org 


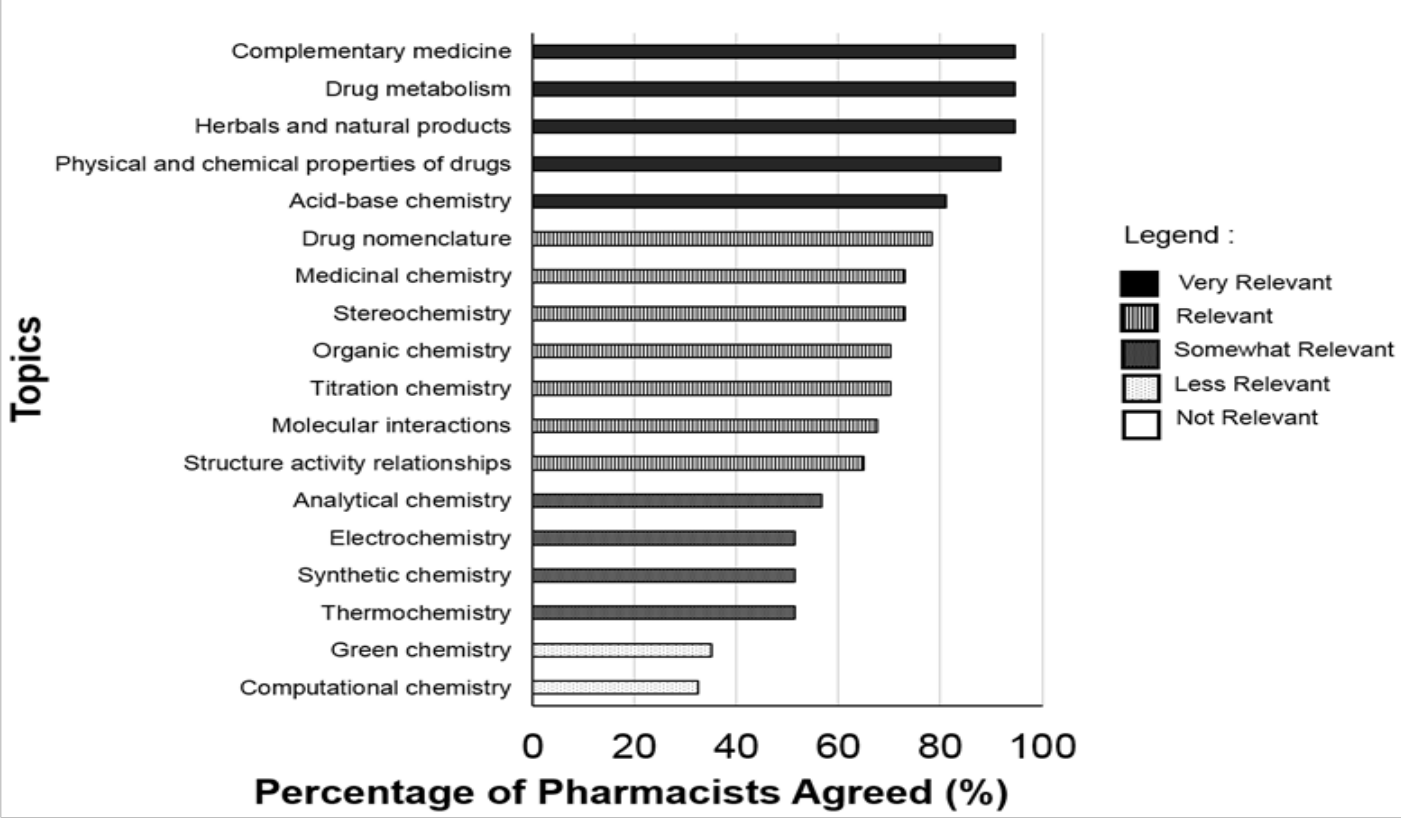

Pictorial Abstract

Percentage of pharmacists agreeing on the relevance of various chemistry topics in their practice. the responses were categorised into Very Relevant (80-100\%), Relevant (60-79\%), Some what Relevant (40-59\%), Less Relevant

(20-39\%) and Not Relevant (0-19\%).

learning environment that is congruent with the outcomes and assessment methods. ${ }^{6}$ As these pharmacists enter the workforce, there has been a constant concern as to whether knowledge gained during their undergraduate study would be essential in nurturing a competent pharmacist.

As a pharmacist, it is a requisite to master the science of chemistry. Conventional module evaluation has failed to identify the relevance of the Chemistry content in developing competent pharmacists because most of modules are formulated as a multi-disciplinary module. For example, "Molecular Interactions" of drugs is emphasised as a sub-chapter of "Pharmacology" along with "Biology" and "Physiology". Therefore, pharmacy schools are facing difficulties in the evaluation of the relevance of Chemistry content as an individual entity within the pharmacy curriculum. To the best of our knowledge, there is no study which was conducted to gather direct feedback from pharmacy graduates regarding the relevance of Chemistry content in the pharmacy curriculum in preparing them for their workplace.

A study published in 2000 showed thirty-three institutions in the United States participated in a national survey related to Chemistry in their Pharmacy Curriculum. ${ }^{7}$ The content of the questionnaire covered twenty-one areas relating to the subject, delving also into delivery methods and teaching styles. Although this study shows that Chemistry is adequately emphasised in the syllabus, it does not show the suitability of the acquired knowl- edge in the practice of pharmacy. The perspectives of the graduates were not included in that study.

The pharmacy curriculum in Malaysia is a four year course which is largely divided into two types: one completed locally in Malaysia while the other is concluded at partner pharmacy schools (United Kingdom, Australia or New Zealand) after two years of theory-based studies in Malaysia. It is also debatable whether the two curricula may have nurtured pharmacists with different levels of competency.

There is no doubt that ongoing curriculum review on pharmacy professionals is essential for the continuous improvement of the profession. Thus the next step would be to conduct a study to determine whether the correct amount of emphasis is given to the more essential aspects of chemistry whilst identifying the other less applicable areas with an option to either offer these as electives or to remove them from the syllabus altogether. Therefore, the current study was aimed at evaluating the relevance of the different aspects of chemistry modules taught in pharmacy schools to the practice of the profession. We were also interested to understand the underlying reasoning behind their comments.

\section{MATERIAL AND METHODS}

This is a cross-sectional, survey-based study where data were collected using self-administered questionnaires distributed online. The survey instrument was modified 
from Roche VF and coworkers, ${ }^{7}$ as well as based on the topics covered in the curriculum of IMU, which is one of the private pharmacy institutions in Malaysia. ${ }^{1} \mathrm{IMU}$ offers pharmacy curricula that are either to be completed entirely in Malaysia [the Bachelor of Pharmacy (BPharm) program] or a twinning program with University of Strathclyde, United Kingdom, [the Master of Pharmacy (MPharm) program]. Graduates from either undergraduate pharmacy program, are registerable with the Malaysia Pharmacy Board post-completion of a one year pharmacy pupillage training. The survey questionnaire was sent to IMU pharmacy alumni of both programs with a minimum of 1 year work experience in their respective fields. There are two types of data that can be derived; quantifiable and qualitative. Respondents were first asked to answer "Yes" or "No" to the question "Is knowledge in (chemistry subject) essential for a competent pharmacist?"; which is quantifiable. Each topic was categorised as very relevant (80-100\%), relevant $(60-79 \%)$, somewhat relevant $(40-59 \%)$, less relevant (20-39\%) and not relevant (0-19\%) according to the average responses from the pharmacists.

Taking into account the wide scope of practice; the pharmacist respondents were divided into four main groups, namely (1) academic, (2) community, (3) hospital, and (4) regulatory. All respondents had voluntarily consented to this study. The academic pharmacists are mainly involved in teaching and research in colleges or universities. This also included those academics currently pursuing postgraduate studies. The second group consisted of community pharmacists practicing in a retail pharmacy setting. The hospital pharmacists made up the third group of respondents. They are involved in the provision of clinical services such as out-patient, in-patient, therapeutic drug monitoring, and evidencebased drug usage in a hospital setting. The fourth group were regulatory pharmacists who are largely involved in enforcement, policy making, or product registration, manufacturing and commercialisation. Responses from each group of pharmacists were also compared in order to understand whether differences in job scope might affect their perceptions.

For each question, the respondents were also requested to elaborate on why they responded this way, which constitutes the qualitative analysis. This gives us further insight into how much they agreed or disagreed with the questions posed. This method of data collection was selected over other number scoring methods in order to discern the subjective perceptions of respondents pertaining to each question. Respondents described their actual experience at work and this was also helpful to guide us in our suggestions for improvement of the current pharmacy curriculum.

In order to assure data integrity and to minimise any potential bias in this study, both quantitative and qualitative data analyses were conducted by three independent investigators who were not respondents to this survey, and not lecturing in any of the IMU BPharm or MPharm curricula. The data were then analysed, compared and compiled by these three investigators. The survey was concluded when the qualitative feedback from respondents had reached saturation, which meant that there was no new comment remaining to be gathered from the respondents. Quantitative results were presented as average \pm standard deviation. Statistical analysis was performed using Student $t$-test or one

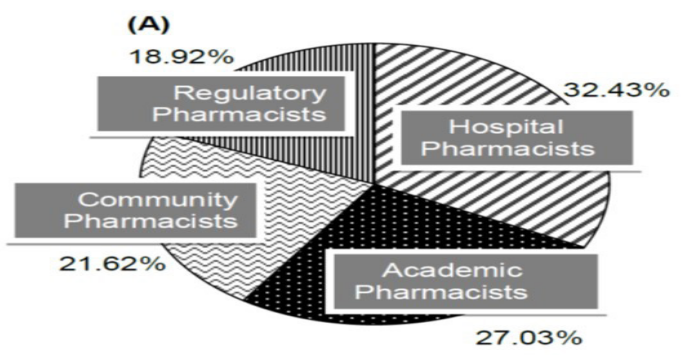

(B)

(C)

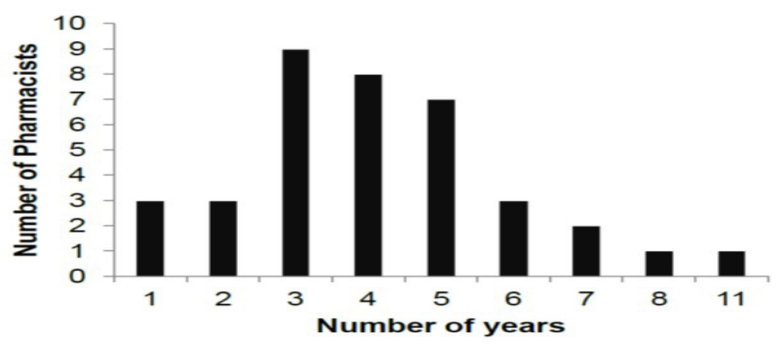

Figure 1: Demographic distrubution of respondents. The surveyed respondents were from four different sectors (A) and from both BPharm and MPharm curricula (B). The distribution of respondents according to the number of years of work experience were also recorded $(\mathrm{C})$ 


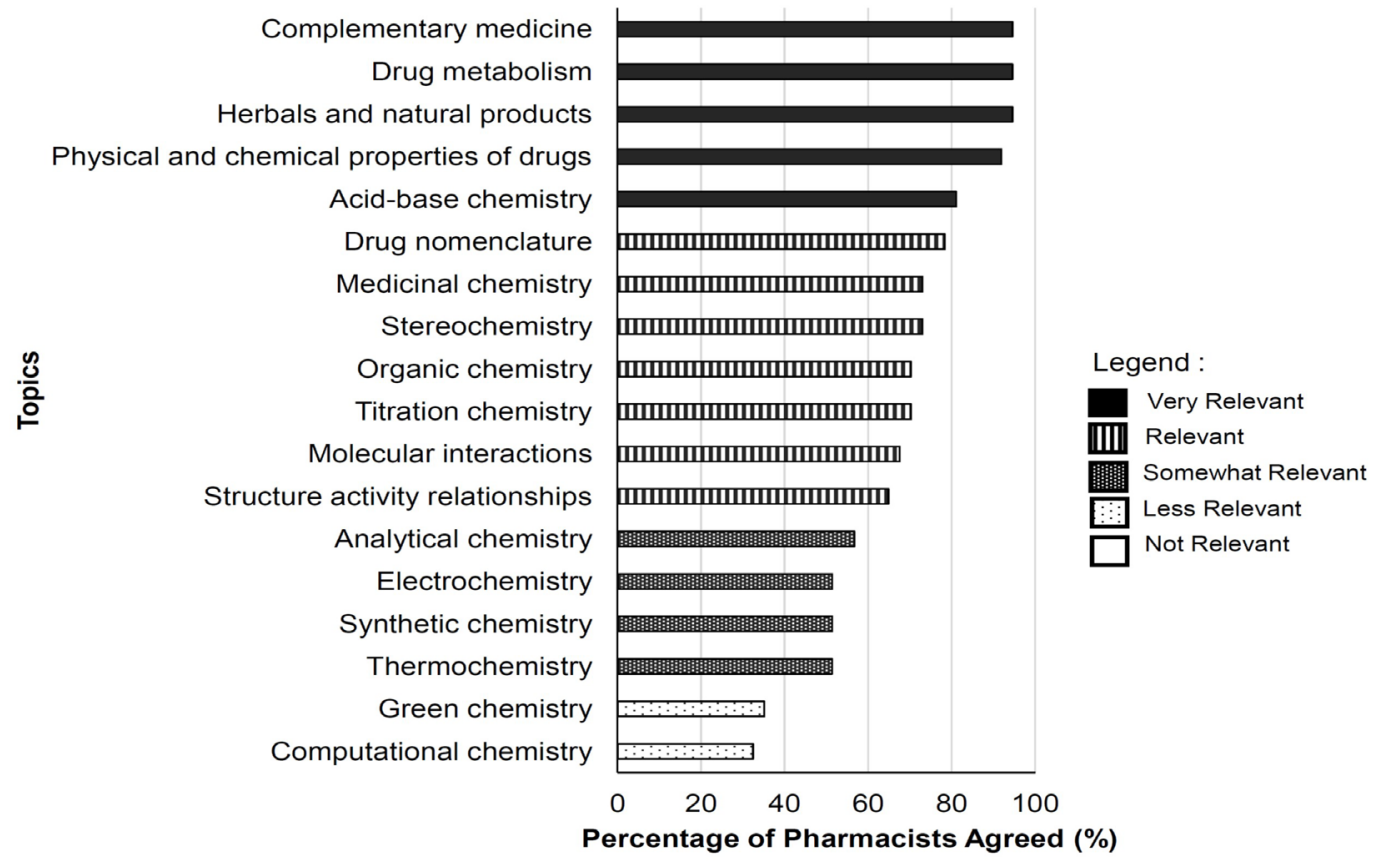

Figure 2: Percentage of pharmacists agreeing on the relevance of various chemistry topics in their practice. The responses were categorised into Very Relevant (80-100\%), Relevant (60-79\%), Somewhat Relevant (40-59\%), Less Relevant (20-39\%) and Not Relevant (0-19\%)

way ANOVA post hoc Least Statistical Difference (LSD) where $* p<0.05, * * p<0.01$, and $* * * p<0.001$ indicated significant statistical difference.

\section{RESULTS AND DISCUSSION}

\section{Socio-demographics}

A total of 37 respondents participated in this study (Figure $1 \mathrm{~A})$, where $7(18.92 \%)$ were regulatory pharmacists; $8(21.62 \%)$ were community pharmacists; $12(32.43 \%)$ were hospital pharmacists; and $10(27.03 \%)$ were academic pharmacists. These pharmacists were either graduates from the Bachelor of Pharmacy (BPharm) program $(54.05 \%)$ or the Master of Pharmacy (MPharm) program $(45.95 \%)$ (Figure 1B). Figure 1C shows the number of years of employment as pharmacists which ranged from 1 to 11 years, with a majority of the respondents having worked for between 3 to 5 years.

\section{Knowledge in most Chemistry topics in pharmacy curriculum is relevant in pharmacy practice.}

As shown in the Figure 2, most of the Chemistry topics in the pharmacy curriculum were rated as very relevant (5/18 topics) or relevant ( $7 / 18$ topics) based on the average responses from the pharmacists. More than 94\% of respondents agreed that knowledge in "Drug metabolism" and "Herbal and natural products" are essential in their practice as pharmacists. The knowledge in "Computational chemistry" and "Green chem- istry" was rated as less relevant with only $32.43 \%$ and $35.13 \%$, respectively, of the total respondents agreeing on their relevance in their practice.

\section{Pharmacy graduates from different programs have similar responses}

Pharmacists who graduated from different programs (BPharm and MPharm) were taught using different curricula based on the guidelines from professional bodies of the respective countries. As shown in Figure 3A, the average percentages of pharmacists agreed in both programmes were not statistically significantly different $(p=0.513)$. It is evident that pharmacists from both BPharm and MPharm programs shared similar views on the importance of Chemistry topics in developing competent pharmacists.

\section{Knowledge in Chemistry may be less relevant to regulatory pharmacists in Malaysia}

Regulatory pharmacists expressed unique views as compared with the other career tracks: hospital $(p<0.001)$, academic $(p=0.003)$ and community $(p<0.001)$ (Figure $3 \mathrm{~B}$ and Figure 4). Only a very low percentage of regulatory pharmacists agreed that knowledge in "Titration Chemistry", "Acid-base Chemistry", "Electrochemistry", "Stereochemistry", "Drug nomenclature", "Molecular interactions", "Physical and chemical properties", "Organic Chemistry", "Medicinal Chemistry", "Synthetic Chemistry", "Structure activity relationships", "Analytical Chemistry" and "Green Chemistry" 
(A)

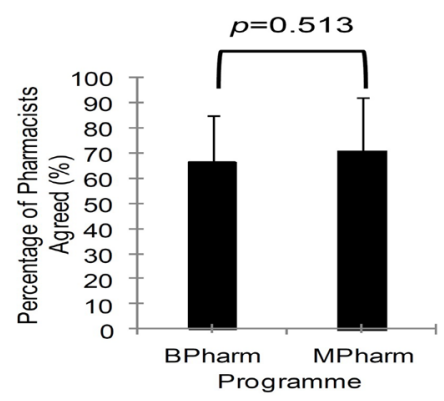

(B)
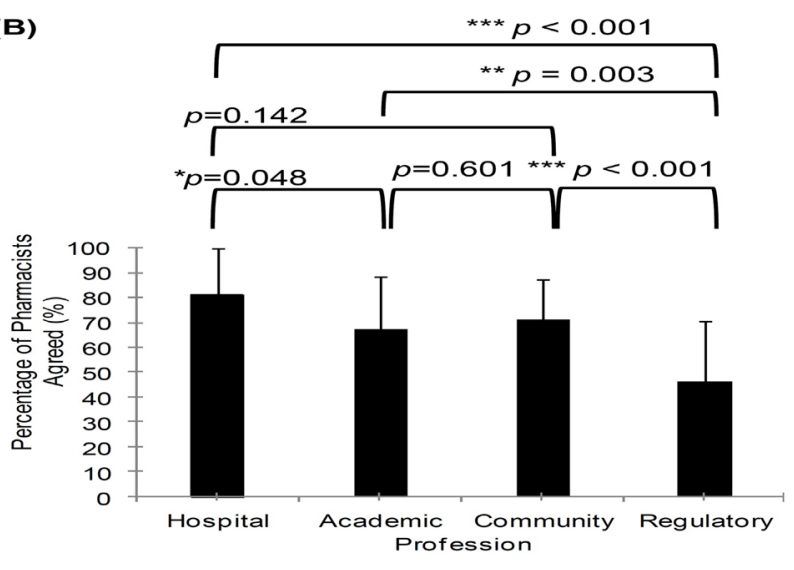

Figure 3: Average percentages of pharmacists agreeing that the topics are essential for a competent pharmacist according to their Pharmacy Curricula (A) and current job scope (B). Bars represent the average \pm standard deviation of the 18 topics. Statistical analysis was performed using Student t-test or one way ANOVA post hoc Least Statistical Difference (LSD) where * $p<0.05$, ${ }^{* \star} p<0.01$, and ${ }^{* \star *} p<0.001$ indicated significant statistical difference.

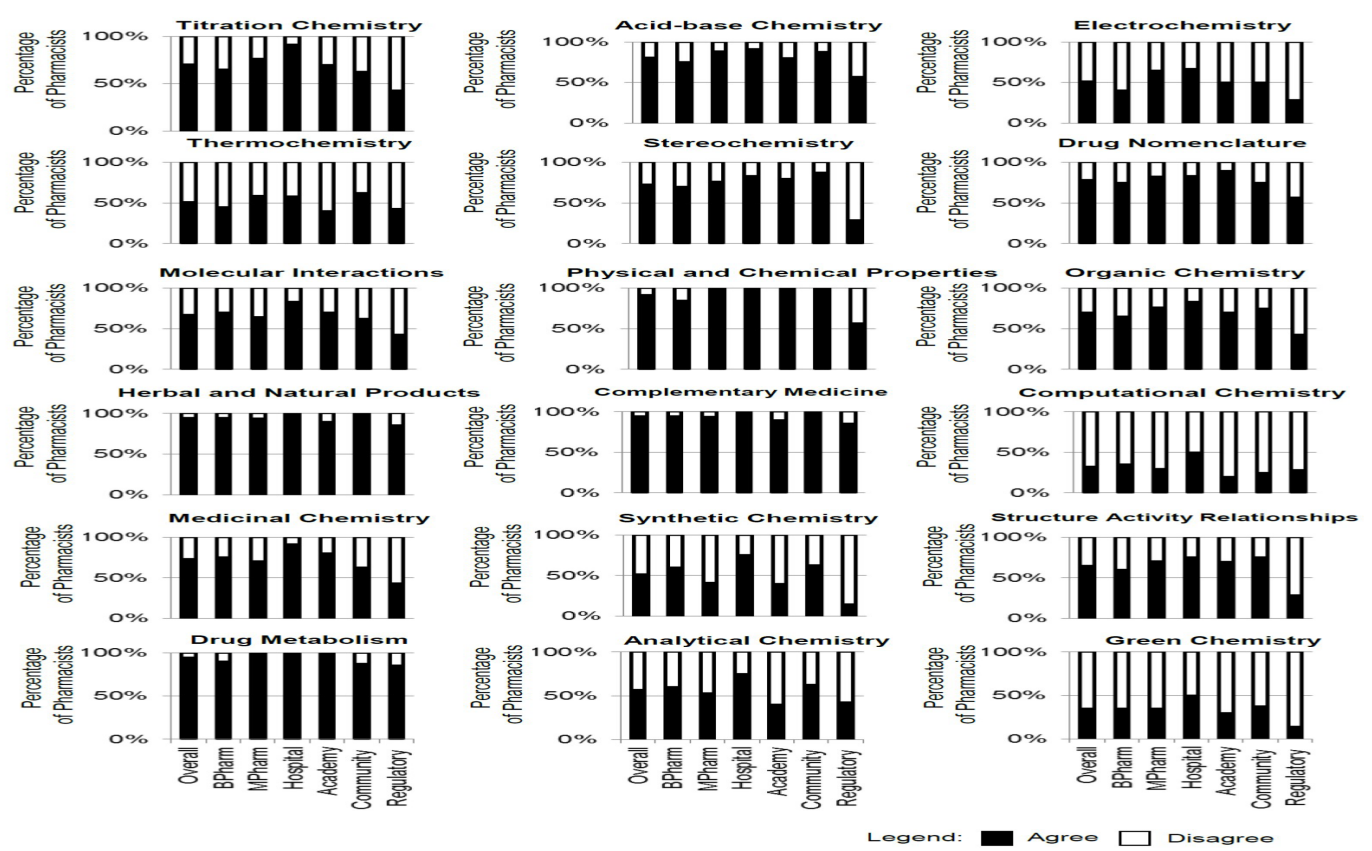

Figure 4: Percentage of pharmacists agreeing/disagreeing on the relevance of individual chemistry topics to their practice

(Figure 4) is relevant to their practice. The regulatory pharmacists commented that:

"(I) have not used it since graduation. A research or industrial pharmacist might need to know the basics, but can always refer to a chemist for help or assistance" (R2; Female; 5 years as regulatory pharmacist)

"No, although (molecular interactions) might be helpful in understanding pharmacology" (R3; Male; 4 years as regulatory pharmacist)
"The knowledge is not used during work unless the pharmacist is in research and development field' (R5; Female; 5 years as regulatory pharmacist)

However, some pharmacists from academia, hospital and community were also of the opinion that knowledge in chemistry is only essential for pharmacists actively involved in research and development, as exemplified by the following responses: 
"No, unless you are analytical or research or academic pharmacists" (C8; Male, 5 years as community pharmacist)

"Not useful except for research" (A7; Female, 7 years as academic pharmacist)

"It might be important to industrial pharmacist." (H1; Female, 3 years as hospital pharmacist)

\section{Chemistry has a pivotal role in developing competent pharmacists}

Despite the differences in the feedback from the different pharmacy professions, there are 5 major reasons why knowledge in Chemistry topics is deemed essential to pharmacy practice. This includes (1) basic pharmacy knowledge, (2) applicability in all settings, (3) understanding the mechanisms of action of drugs, (4) drug discovery and (5) patient education and counselling. Each of the reasons is discussed as follows:

\section{Basic Pharmacy Knowledge}

The main reason why most of the chemistry topics were regarded as essential by most of the respondents was that it was the requisite knowledge for pharmacists as exemplified by the following responses:

"Yes, that is the basic chemistry knowledge that every competent pharmacist should be equipped with." (A4; Female, 4 years as academic pharmacist)

"Yes, in terms of basic knowledge." (A8; Female, 3 years as academic pharmacist)

"Yes. For basic understanding of chemistry and pharmacology” (H10; Male, 8 years as hospital pharmacist)

"Yes, basic knowledge that we need to know to progress to more advanced subjects of the study." (H11; Female, 3 years as hospital pharmacist)

"Every pharmacist needs to know this. This is the most basic knowledge a pharmacist should have." (R2; Female, 5 years as regulatory pharmacist)

"Yes. A strong foundation in chemistry is essential for any competent pharmacist." (R6; Female, 4 years as regulatory pharmacist)

"Yes; for basic foundation in understanding the energy and heat associated with chemical reactions and/ or physical transformations. (C6; Female, 3 years as community pharmacist)

\section{Applicable in All Settings}

Many pharmacists also commented that a knowledge of Chemistry is useful in all pharmacy settings.

"Very important and applicable in all settings." (A2; Female, 2 years as academic pharmacist)
"Yes. Much applicable in various pharmacy setting." (H4; Female, 4 years as hospital pharmacist)

"Yes. It's important for pharmacists in industry setting, and also those in academia. In some degree, for pharmacists in clinical setting, both hospital and community to judge the efficacy of some new drugs in the market." (C2; Male, 6 years as community pharmacist)

\section{Understanding the mechanism of actions of drugs}

The pharmacy curriculum stresses the mechanisms of action of drugs/medicines during the pre-clinical and clinical years. Pharmacists commented that knowledge in Chemistry was regarded as essential as it helps the pharmacists to provide the best services in all settings. It is not surprising to observe such responses, because in the pharmacy curriculum, in Medicinal Chemistry modules, pharmacy students will be learning the mechanisms of action of drugs, which provides the foundation for pharmacists to make optimal patient(or client)-specific therapeutic decisions. ${ }^{8}$

"I find it useful to understand this, to know how changes in molecules itself would affect the activity of a drug. In a way it is like learning history, about how certain drugs became how they are now." (A5; Female, 2 years as academic pharmacist)

"Yes. To understand the reason behind the drug's activity." (A7; Female, 7 years as academic pharmacist)

"Yes. It has relation to how drug works. So, pharmacists need to know." (A8; Female, 3 years as academic pharmacist)

"Yes. It's because without knowledge in stereochemistry, one would not understand why a different cbiral molecule exerts different receptor response due to spatial differences." (C8; Male, 4 years as community pharmacist)

"It is important as it relates to the processes in compounding and interactions between drugs." (H1; Female, 3 years as hospital pharmacist)

"Yes, because modification of a chemical group of a drug compound would bring different result and this is important to study the effectiveness in terms of studying selectivity and pharmacokinetics of the drug." (H8; Female, 4 years as hospital pharmacist)

"Yes; for understanding of the characteristics of the ensyme reactions that inactivates the drug, the relationship with elimination process and thereby for dosage adjustment if necessary." (C6; Female, 3 years as community pharmacist)

"From there, you would know how the drug works and which organs are used to metabolise the drug. If the liver is used to metabolise the drug and the patient has liver impairment, dose 
adjustment to the drug or strict contraindication is necessary."

(R5; Female, 5 years as regulatory pharmacist)

\section{Research and drug discovery}

Some pharmacists highlighted that knowledge in Chemistry has driven the research and development in pharmacy. It is particularly important for those involved in drug discovery. This comment has been consistent throughout all sectors. Drug discovery has always been an indispensable component in pharmacy curricula due to the nature and scope of the job. ${ }^{8}$

"It is important for drug discovery." (A1; Male, 6 years as academic pharmacist)

"Yes. It is useful for those who will be involved in drug discovery and design." (A6; Female, 3 years as academic pharmacist)

"I think this is important for pharmacy students who venture into drug discovery research to have this knowledge." (A9; Female, 11 years as academic pharmacist)

"Useful for pharmacist in drug discovery." (H7; Female, 4 years as hospital pharmacist)

"For drug discovery and pharmacentical field, I believe it is essential'. (C5; Male, 3 years as community pharmacist)

"Yes; for advancing productivity in research and drug discovery process." (C6; Female, 3 years as community pharmacist)

"Yes. It is important in terms of research and drug discovery." (C7; Female, 7 years as community pharmacist)

"Mostly used for research." (R2; Female, 5 years as regulatory pharmacist)

\section{Patient Education and Counselling}

Community, hospital and even academic pharmacists can apply/use their knowledge acquired from Chemistry modules in providing effective patient education and counselling.

"Yes. A competent pharmacist should know how to justify the use of complementary medicine and hence provide proper advice and counselling to consumers." (A6; Female, 3 years as academic pharmacist)

"Yes. Important to understand the interactions so that they can explain to the patients when asked." (A7; Female, 7 years as academic pharmacist)

"Yes, especially evidence-based knowledge as it will enable pharmacist to advice patients appropriately." (C7; Female, 7 years as community pharmacist)

"Yes because a pharmacist should be able to give thorough counselling on the available products and their interaction with the medication(s) the patients are taking." (H7; Female, 4 years as hospital pharmacist)

"Yes. Sometimes patients ask for our advice as they would like to try therapies other than what has been recommended by the doctor." (H9; Female, 5 years as hospital pharmacist) This study only canvassed pharmacy graduates of the International Medical University, so it may not be representative for graduates from all Malaysian institutions offering a Pharmacy Degree. Furthermore, the content of each institution's learning modules may very well differ despite them having similar curricular structures. This in turn may affect the student's mastery and ability to apply the knowledge gained during their degree in their chosen job sector. A broader respondent base would therefore be needed to cover the curricula of various institutions. However, this survey seems to provide a good preliminary set of data to warrant further curriculum development. This study also only aimed to identify the aspects of chemistry relevant to the various pharmaceutical working environments/sectors. The study did not attempt to rate these subjects in any order of relevance to the job scope. These data are important so that the Pharmacy curricula may be altered to place emphasis on the most relevant subjects and perhaps explore the subject in greater depth. A deeper understanding of the key Chemistry subjects will definitely ensure a higher level of competency amongst pharmacists in every sector.

\section{CONCLUSION AND RECOMMENDATIONS}

Pharmacists operate in diverse employment sectors with different duties and, inter alia, it is clear that knowledge in the subjects of Chemistry is a major requirement for them to perform their daily tasks. This study concludes that a majority of the components in the current chemistry aspects of the IMU Pharmacy degree programs are still essential and relevant in developing competent pharmacists in their chosen discipline. Therefore, the current curriculum for Chemistry should be refined so as to ensure the competency of graduates. The results of this study as well as similar ones in the future will be used as a guide during curriculum reviews for the continuous improvement of the university's standards of education.

\section{ACKNOWLEDGEMENT}

The authors would like to acknowledge all the pharmacists who took part in this study.

\section{ABBREVIATIONS}

BPharm: Bachelor of Pharmacy ;IMU: International Medical University ; LSD: Least Statistical Difference ; MPharm: Master of Pharmacy 


\section{SUMMARY}

- Knowledge in most Chemistry topics in pharmacycurriculum is relevant in pharmacy practice.

- Pharmacy graduates from different programs havesimilar responses.

- Knowledge in Chemistry may be less relevant toregulatory pharmacists in Malaysia.

- Chemistry has a pivotal role in developing competentpharmacists.

\section{About Authors}

Dr. Chun Wai Mai: currently working as Lecturer in Department of Pharmaceutical Chemistry, International Medical University. He is a research fellow with British Antartic Survey, Cambridge, United Kingdom and was a research fellow with Strathclyde Institute of Pharmacy and Biomedical Sciences, University of Strathclyde, United Kingdom. He is currently a registered pharmacist in Malaysia. He has secured various research grants and his team has been actively publishing in numerous peer review journals, conference proceedings and newspapers pertaining todiscovery of biomarkers, drug discovery, and pharmaceutical education.

\section{REFERENCES}

1. Kolluru S. An active-learning assignment requiring pharmacy students to write medicinal chemistry examination questions. Am J Pharm Educ. 2012; 76(6): 112.

2. El Sayed KA, Chelette CT. Laboratory exercises to teach clinically relevant chemistry of antibiotics. Am J Pharm Educ. 2014; 78(2): 37.

3. Conway SE, Smith WJ, Truong TH, Shadid J. Interprofessional pharmacy observation activity for third-year dental students. J Dent Educ. 2014; 78(9): 1313-8.

4. Richardson A, Bracegirdle L, McLachlan SI, Chapman SR. Use of a threedimensional virtual environment to teach drug-receptor interactions. Am J Pharm Educ. 2013; 77(1): 11.
5. Shah S, Lynch LM, Macias-Moriarity LZ. Crossword puzzles as a tool to enhance learning about anti-ulcer agents. Am J Pharm Educ. 2010; 74(7): 117.

6. Beck DE. Outcomes and experiential education. Pharmacotherapy 2000; 20(10p2): 297S-306S.

7. Roche VF, Davis PJ, Pankaskie MC, Currie BL, Roche EB, Sindelar RD, et al. The Status of Chemistry Content in the Professional Pharmacy Curriculum: Results of a National Survey (Galley). Am J Pharm Educ. 2000; 64(1): 239-51.

8. Khan MO, Deimling MJ, Philip A. Medicinal chemistry and the pharmacy curriculum. Am J Pharm Educ. 2011 Oct 10; 75(8): 161. 